\title{
$\Im \mathfrak{n} \mathfrak{b} \mathfrak{t}$
}

KOГEОMПAz: fragmente úber apolalypt. B̧e: beimnifie • • • • • • 3wey Ëjerflein zur neueften beutjijen sitteratur -23 Becenfion ber Eritil ber reinen Bernunft. . - 45 Briefe von 1779 bis 1784 . $\quad$ • $~-~-55$ 
$\mathfrak{h} \mathfrak{a} \mathfrak{m} \mathfrak{a} \mathfrak{n} \mathfrak{n}^{\prime} \mathfrak{z}$

(b) $\mathfrak{x} \mathfrak{i} \mathfrak{f} \mathfrak{t} \mathfrak{e} \mathfrak{n}$.

fgeraus gegeben

von

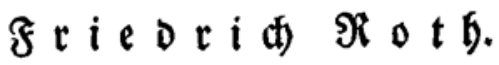

Sed d ter

Berlin,

bey ए. Reimcr 1824 . 
